\title{
PD-L1 Status Unknown
}

National Cancer Institute

\section{Source}

National Cancer Institute. PD-L1 Status Unknown. NCI Thesaurus. Code C122810.

Indicates that the immunohistochemical test result for PD-L1 is unknown or the test has not been performed. 